\title{
Supplementary materials: A Robust calibration/validation protocol of a hydrological model using hidden Markov states
}

Etienne Guilpart ${ }^{1}$, Vahid Espanmanesh ${ }^{1}$, Amaury Tilmant ${ }^{1}$, and François Anctil ${ }^{1}$

${ }^{1}$ Département de génie civil et de génie des eaux, Université Laval, Québec, Canada

Correspondence: E. Guilpart (etienne.guilpart@gmail.com)

S1 Calibrations and validations on the wet historical episode $T^{1945-1971}$

Here, we apply our protocol on the period 1945-1971 $\left(T^{1945-1971}\right)$, which can be consider as a wet historical episode of the SRB. The results are displayed in Figure S1, Table S1 and Table S2.

The wet historical episode $T^{1945-1971}$

\begin{tabular}{|c|c|c|c|c|c|c|c|}
\hline \multirow{4}{*}{ Pettitt test } & Basins & $\mathrm{p}$ value & \multicolumn{5}{|l|}{ Year break } \\
\hline & Daka Saidou & 0.694 & \multicolumn{5}{|l|}{ - } \\
\hline & Oualia & 0.399 & \multicolumn{5}{|l|}{ - } \\
\hline & Bakel & 0.646 & \multicolumn{5}{|l|}{-} \\
\hline \multirow{7}{*}{ 2-states-HMM } & Basins & $\mu$ & $\sigma$ & $\delta$ & M & & \\
\hline & \multirow[t]{2}{*}{ Daka Saidou } & \multirow{2}{*}{$366 ; 273.3$} & \multirow{2}{*}{$25.6 ; 43.6$} & \multirow{2}{*}{1,0} & 0.864 & 0.136 & \\
\hline & & & & & 0.413 & 0.587 & \\
\hline & \multirow{2}{*}{ Oualia } & \multirow{2}{*}{$201.8 ; 124$} & \multirow{2}{*}{$32.1 ; 15$} & \multirow{2}{*}{0,1} & 0.344 & 0.656 & \\
\hline & & & & & 0.287 & 0.713 & \\
\hline & \multirow{2}{*}{ Bakel } & \multirow{2}{*}{$1073 ; 713$} & \multirow{2}{*}{$65.4 ; 125.4$} & \multirow{2}{*}{0,1} & 0.684 & 0.316 & \\
\hline & & & & & 0.535 & 0.465 & \\
\hline \multirow{9}{*}{ 3-states-HMM } & \multirow{4}{*}{ Daka Saidou } & \multirow{3}{*}{$361.6 ; 289.8 ; 217$} & \multirow{3}{*}{$15.3 ; 20.9 ; 27.2$} & \multirow{3}{*}{$0,1,0$} & 0.589 & 0.158 & 0.252 \\
\hline & & & & & 0.141 & 0.696 & 0.163 \\
\hline & & & & & 0.154 & 0.252 & 0.594 \\
\hline & & \multirow{3}{*}{$127.7 ; 192.9 ; 243.9$} & \multirow{3}{*}{$17 ; 19.5 ; 6.5$} & \multirow{3}{*}{$0,1,1$} & 0.397 & 0.281 & 0.322 \\
\hline & \multirow[t]{3}{*}{ Oualia } & & & & 0.482 & 0.435 & 0.083 \\
\hline & & & & & 0 & 0.835 & 0.165 \\
\hline & & \multirow{3}{*}{$243.9 ; 192.9 ; 127.7$} & \multirow{3}{*}{$6.5 ; 19.5 ; 17$} & \multirow{3}{*}{$0,1,0$} & 0.397 & 0.281 & 0.322 \\
\hline & \multirow[t]{2}{*}{ Bakel } & & & & 0.482 & 0.435 & 0.083 \\
\hline & & & & & 0 & 0.835 & 0.165 \\
\hline
\end{tabular}

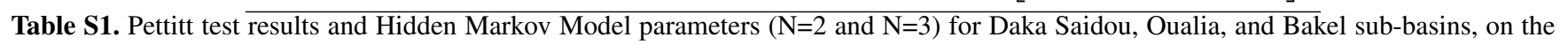
wet sub-sequence $T^{1945-1971}$.

For the three sub-basins, Pettitt's tests are inconclusive, meaning that there is no trend in those sub-sequences. The transition 5 probability matrices for the 2-states HMM and 3-states HMM are diverging from an identity matrix, indicating that the temporal persistence is less pronounced. However, in a such situation, HMM classification remains a useful tool to divide a period 


\section{The wet sub-sequence $T^{1945-1971}$}
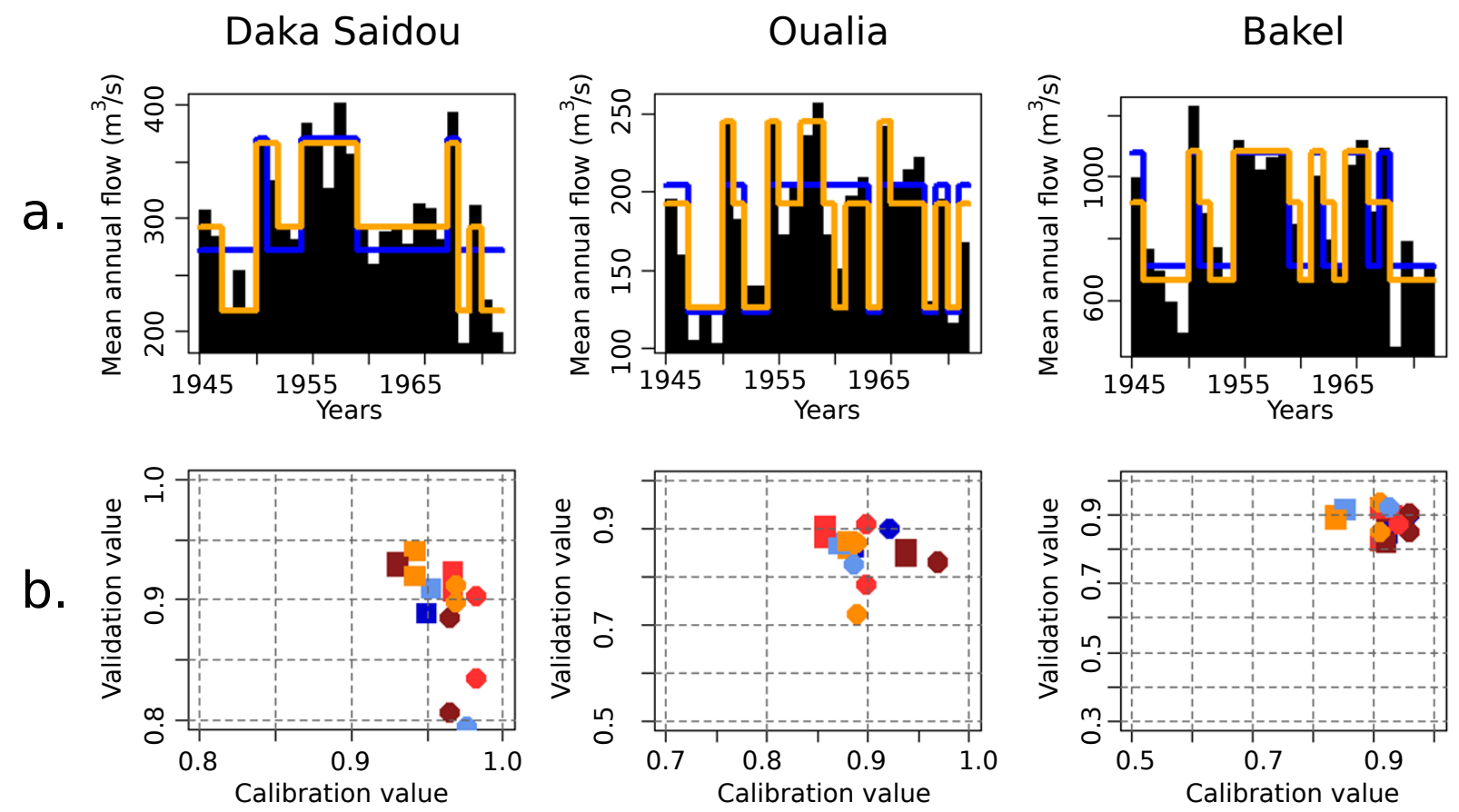

Figure S1. a. Years classifications of $T^{1945-1971}$ according to the Pettitt test (vertical red lines), 2-states-HMM (in blue) and 3-states-HMM (in orange); b. Scatter-plot of NSE (squares) and KGE (dots) calibration/validation values. Light green refers to the case 1 (Pettitt test, calibration on $T_{\text {pettitt.wet }}$ and validation on $T_{\text {pettitt.dry }}$ ); dark green to the case 2(Pettitt test, calibration on $T_{p e t t i t t . d r y}$ and validation on $T_{\text {pettitt.wet }}$ ); Light blue to the case 3 (2-states-HMM, calibration on $T_{2 H M M . d r y}$ and validation on $T_{2 H M M . w e t}$ ), and dark blue to the opposite (case 4); Orange to the case 5 (3-states-HMM, calibration on $T_{3 H M M . d r y}$ and validation on $T_{3 H M M . n o r}$ and $T_{3 H M M . w e t}$ ); red to the case 6 , and dark red to the seventh case.

into climate sub-sequences, and thus to apply a protocol following the differential split-sample test.

With no surprise, we note that the mean annual flow of dry sub-sequences $\left(T_{2 H M M . d r y}^{1945-1971}\right.$ and $\left.T_{3 H M M}^{1945-1971}\right)$ are relatively high (in comparison with $T_{2 H M M \text {.dry }}^{1940-1998}$ and $T_{3 H M M \text {.dry }}^{1940-1998}$ ). We note that the HMM could provide a year classification in which the number of years is very small (for example: $T_{3 H M M M . w e t}^{1945-1971}$ for Oualia (5 years)).

In addition, transition probabilities values indicate that climate states for $T_{1945-1971}$ are less well distinct (close to 0.5 ) than for $T_{1940-1998}$ (close to 1 or 0$)$. 
Daka Saidou

The wet sub-sequence $T^{1945-1971}$

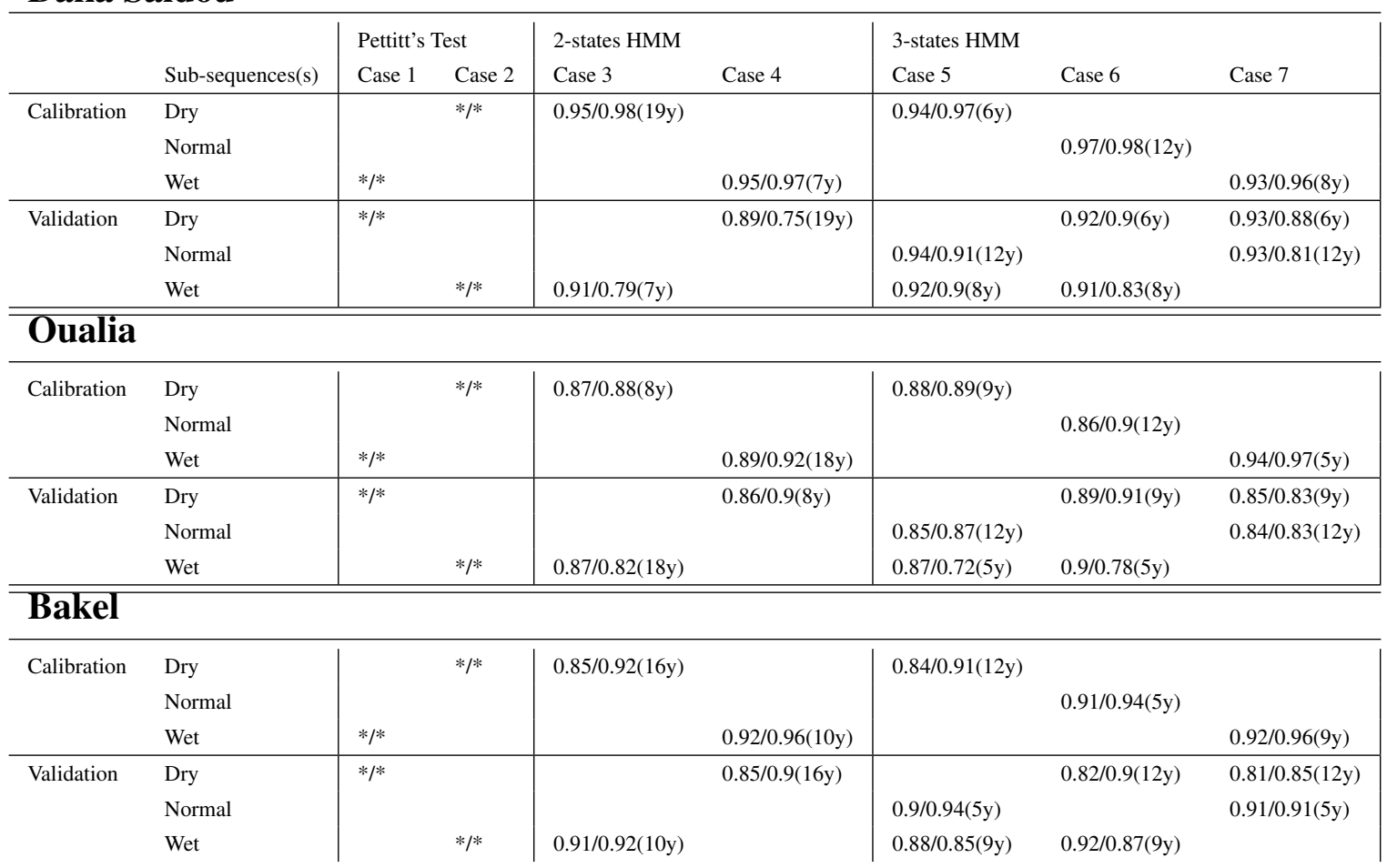

Table S2. Table of NSE/KGE calibration and validation scores according to the seven cases for the wet sub-sequence $T^{1945-1971}$. As the Pettitt's test is not conclusive here, no calibration/validation scores are given (symbols $* / *$ ).

\section{S2 Calibrations and validations on the dry historical episode $T^{1972-1998}$}

Here, we apply our protocol on the period 1972-1998 $\left(T^{1972-1998}\right)$, which can be consider as a dry historical episode of the SRB. The results are displayed in Figure S2, Table S3 and Table S4.

Likewise in S1, there is no clear trend and poor p-values are found with the Pettitt test while the examination of the transition probability matrices reveal a lower temporal persistence compared to the cases with the entire streamflow record.

Here, we are in the opposite situation than before: the wet states $T_{2 H \text { M M.wet }}^{1972-1998}$ and $T_{3 H \text { M M M.wet }}^{1972-1998}$ have relatively dry mean annual flows. Again, the HMM classification could yield to a number of years too small in some situations (example: $T_{3 H M M M . d r y}^{1972-1998}$ for Daka Saidou (5 years), $T_{3 H M M M . n o r}^{1972-1998}$ for Oualia (5 years)). This could constitute an issue for the calibration or the validation phase. These two points are addressed in the discussion section. Similarly to $T_{1945-1971}$, transition probabilities values indicate that climate states for $T_{1972-1998}$ are less well distinct (close to 0.5 ) than for $T_{1940-1998}$ (close to 1 or 0 ). 
The dry historical episode $T^{1972-1998}$

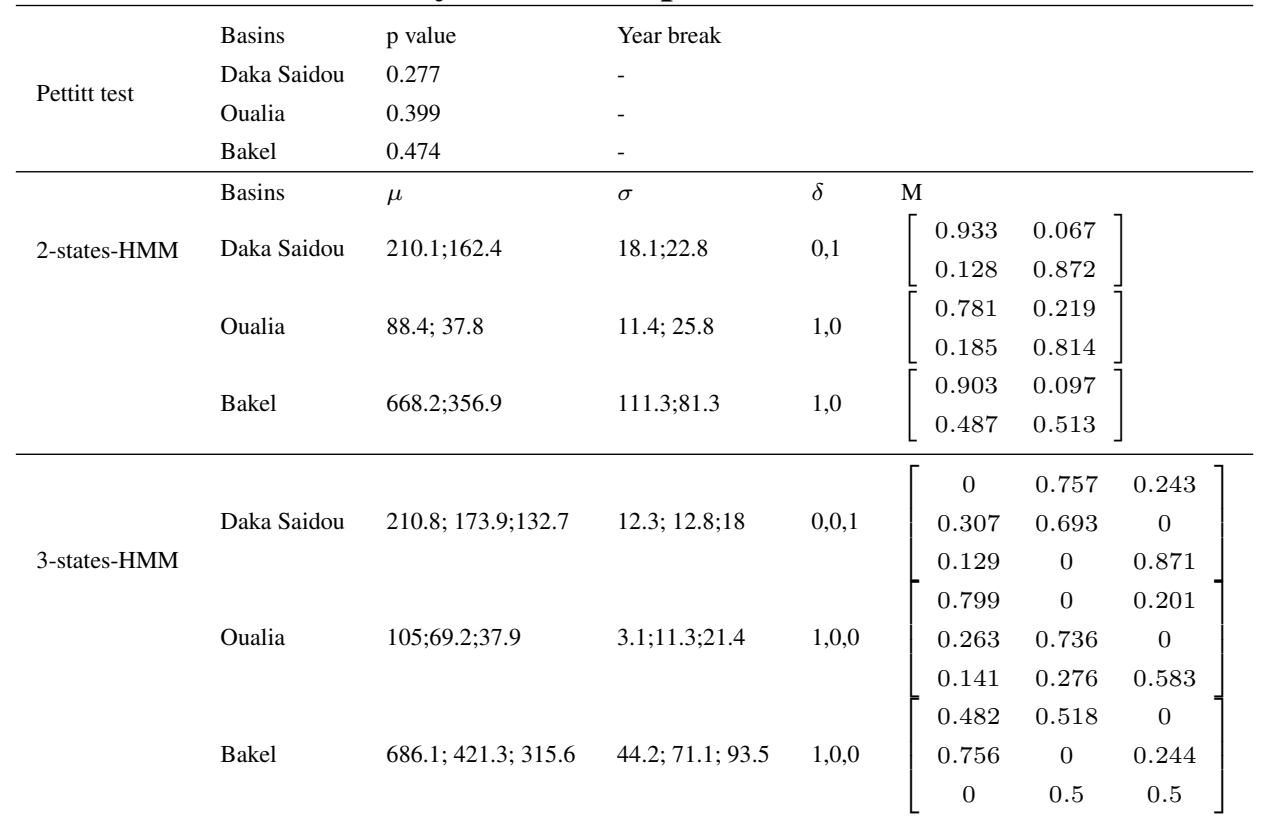

Table S3. Pettitt test results and Hidden Markov Model parameters (N=2 and N=3) for Daka Saidou, Oualia, and Bakel sub-basins, on the dry sub-sequence $T^{1972-1998}$. 
The dry sub-sequence $T^{1972-1998}$
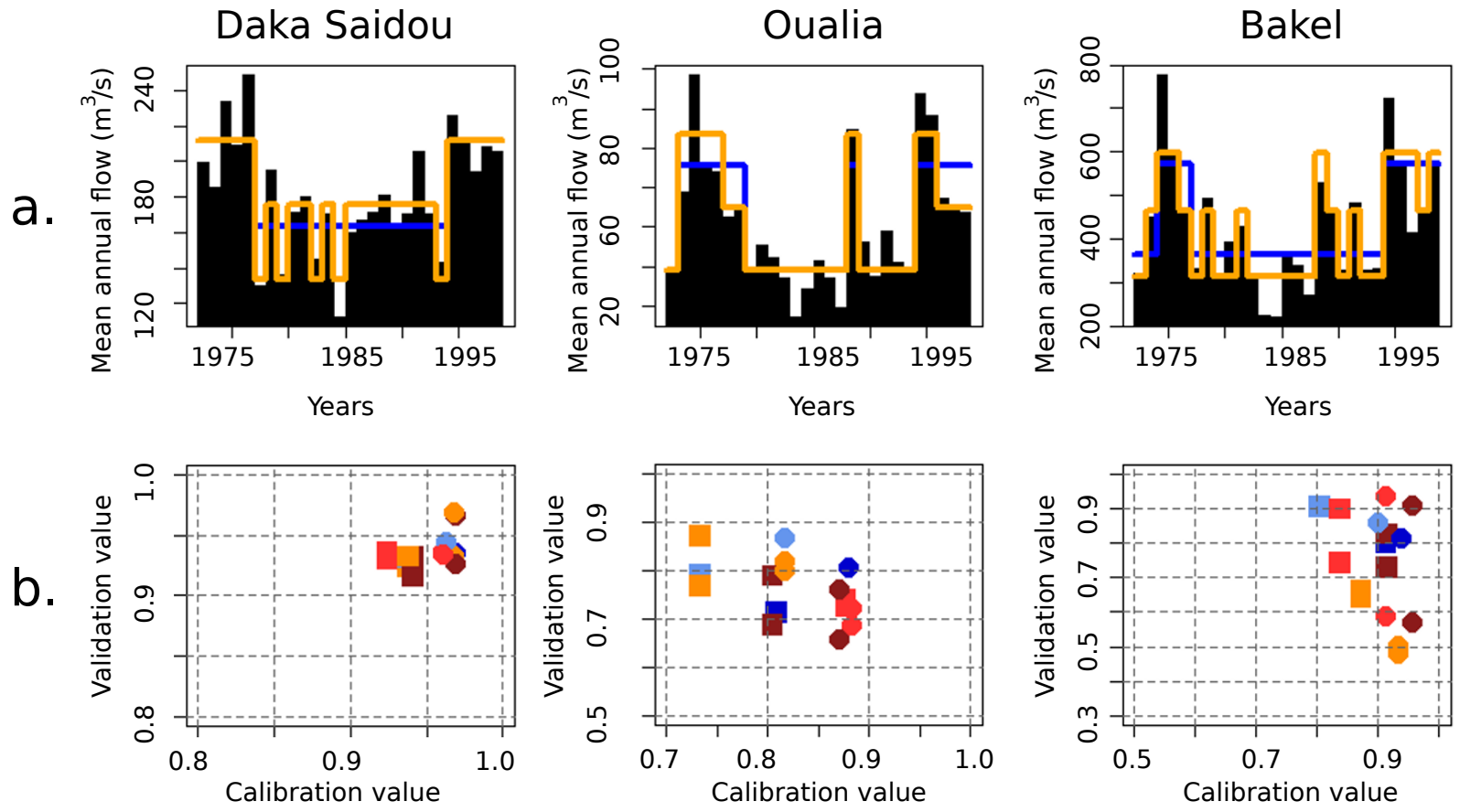

Figure S2. Same than Figure S1, but for $T^{1972-1998}$. 
The dry sub-sequence $T^{1972-1998}$

\begin{tabular}{|c|c|c|c|c|c|c|c|c|}
\hline \multicolumn{9}{|c|}{ Daka Saidou } \\
\hline & & Pettitt's & & 2-states HMM & & 3-states HMM & & \\
\hline & Sub-sequence(s) & Case 1 & Case 2 & Case 3 & Case 4 & Case 5 & Case 6 & Case 7 \\
\hline \multirow[t]{3}{*}{ Calibration } & Dry & & $* / *$ & $0.93 / 0.96(17 \mathrm{y})$ & & $0.94 / 0.97(5 y)$ & & \\
\hline & Normal & & & & & & $0.92 / 0.96(12 y)$ & \\
\hline & Wet & $* / *$ & & & $0.94 / 0.97(9 \mathrm{y})$ & & & $0.94 / 0.97(9 \mathrm{y})$ \\
\hline \multirow[t]{3}{*}{ Validation } & Dry & $* / *$ & & & $0.92 / 0.93(17 \mathrm{y})$ & & $0.94 / 0.94(5 y)$ & $0.93 / 0.97(5 \mathrm{y})$ \\
\hline & Normal & & & & & $0.92 / 0.93(12 y)$ & & $0.93 / 0.81(12 \mathrm{y})$ \\
\hline & Wet & & $* / *$ & $0.93 / 0.94(9 \mathrm{y})$ & & $0.93 / 0.97(9 \mathrm{y})$ & $0.93 / 0.93(9 \mathrm{y})$ & \\
\hline \multicolumn{9}{|l|}{ Oualia } \\
\hline \multirow{3}{*}{ Calibration } & Dry & & $* / *$ & $0.73 / 0.82(14 y)$ & & $0.73 / 0.82(14 y)$ & & \\
\hline & Normal & & & & & & $0.88 / 0.88(5 y)$ & \\
\hline & Wet & $* / *$ & & & $0.81 / 0.88(12 y)$ & & & $0.8 / 0.87(7 \mathrm{y})$ \\
\hline \multirow[t]{3}{*}{ Validation } & Dry & $* / *$ & & & $0.71 / 0.8(14 y)$ & & $0.72 / 0.72(14 \mathrm{y})$ & $0.69 / 0.76(14 y)$ \\
\hline & Normal & & & & & $0.87 / 0.8(5 y)$ & & $0.79 / 0.66(5 y)$ \\
\hline & Wet & & $* *$ & $0.79 / 0.87(12 y)$ & & $0.77 / 0.82(7 y)$ & $0.72 / 0.68(7 \mathrm{y})$ & \\
\hline \multicolumn{9}{|l|}{ Bakel } \\
\hline \multirow[t]{3}{*}{ Calibration } & Dry & & $* *$ & $0.73 / 0.82(18 \mathrm{y})$ & & $0.73 / 0.82(12 y)$ & & \\
\hline & Normal & & & & & & $0.88 / 0.88(7 y)$ & \\
\hline & Wet & $* / *$ & & & $0.81 / 0.88(8 \mathrm{y})$ & & & $0.8 / 0.87(7 \mathrm{y})$ \\
\hline \multirow[t]{3}{*}{ Validation } & Dry & $* / *$ & & & $0.71 / 0.8(18 \mathrm{y})$ & & $0.72 / 0.72(12 \mathrm{y})$ & $0.69 / 0.76(12 \mathrm{y})$ \\
\hline & Normal & & & & & $0.87 / 0.8(7 \mathrm{y})$ & & $0.79 / 0.66(7 \mathrm{y})$ \\
\hline & Wet & & $* / *$ & $0.79 / 0.87(8 \mathrm{y})$ & & $0.77 / 0.82(7 y)$ & $0.74 / 0.68(7 y)$ & \\
\hline
\end{tabular}

Table S4. Table of NSE/KGE calibration and validation scores according to the seven cases for the dry sub-sequence $T^{1972-1998}$. As the Pettitt's test is not conclusive here, no calibration/validation scores are given (symbols $* / *$ ). 